\title{
Una visión sobre los principios de la sustentabilidad para la enseñanza de la arquitectura
}

Jonathan Hammurabi González Lugo

\section{Resumen}

$\mathrm{E}$ 1 presente trabajo aborda una visión para la implementación real y práctica de los principios de la sustentabilidad en la enseñanza de la arquitectura, abordando, en primera instancia, una aproximación al concepto, así como los elementos y aspectos que la determinan. En seguida, se plantea la relación del término de sustentabilidad con la arquitectura y los procesos de enseñanza en las instituciones de nivel superior, aportando algunas apreciaciones que involucren de manera más comprometida a los estudiantes en la búsqueda de la sustentabilidad desde su disciplina y quehacer cotidiano.

Palabras clave: sustentabilidad, diseño arquitectónico, educación universitaria, arquitectura sustentable.

\section{Introducción}

Actualmente, la carrera de arquitectura de la Universidad Autónoma de Aguascalientes, a través del portal web del Centro de Ciencias del Diseño y de la Construcción, muestra en su inicio, como parte de la oferta educativa de la licenciatura, el objetivo de su plan de estudios, que dice textualmente:

Formar un profesional de la arquitectura en los ámbitos del Diseño y la Edificación de espacios habitables desde lo local hasta lo global, con una orientación humanista sensible a los problemas del entorno y capacidad para interpretar los factores socio-culturales y tecnológicos a efectos de mejorar la calidad de vida con base en criterios sustentables (UAA, 2018).

Posteriormente, en el perfil de egreso se asienta la necesidad de mostrar habilidades y conocimientos en la integración de criterios de sustentabilidad en los procesos para la arquitectura dentro de los ámbitos de diseño arquitectónico y edificación de espacios habitables. Lo anterior representa un esfuerzo por alinearse a las tendencias internacionales en torno al cuidado del medio ambiente, y acorde a los objetivos de desarrollo del milenio de la Organización de las Naciones Unidas en su punto número siete, que busca "garantizar la sostenibilidad del medio ambiente" (onu, 2018).

A pesar de lo expuesto, al ser un concepto aplicable a múltiples dimensiones y disciplinas, no existe un solo criterio para su implementación, y faltaría precisar los elementos a evaluar en torno a este tema por parte de los docentes de la carrera de arquitectura y aquellas otras que se relacionen con el desarrollo urbano, territorial y la edificación, tema que aborda el presente artículo. Para ello, es necesario conocer y comprender las implicaciones y variables que involucran la sustentabilidad.

\section{La sustentabilidad}

De acuerdo a la Secretaría de Desarrollo Agrario, Territorial y Urbano (SEDATU, 2014: 14), la sustentabilidad "es un ejercicio destinado a garantizar la reproducción de los recursos naturales para las generaciones futuras, en vista a lograr una capacidad de proseguir y renovar las condiciones sociales, económicas, culturales, políticas y ambientales". Lo anterior se puede explicar de una mejor manera desde lo que los estudiosos en la materia definen como "los tres pilares del desarrollo sustentable": social, económico y ambiental, que se describen en el Esquema 1, a patir de un estudio elaborado por el Programa de las Naciones Unidas para el Medio Ambiente (UNEP, por sus siglas en inglés).

«Un factor que advierte los efectos de entornos urbano-arquitectónicos alejados de principios de sustentabilidad, es el cambio climático.»》 
Esquema 1. Los tres pilares del desarrollo sustentable

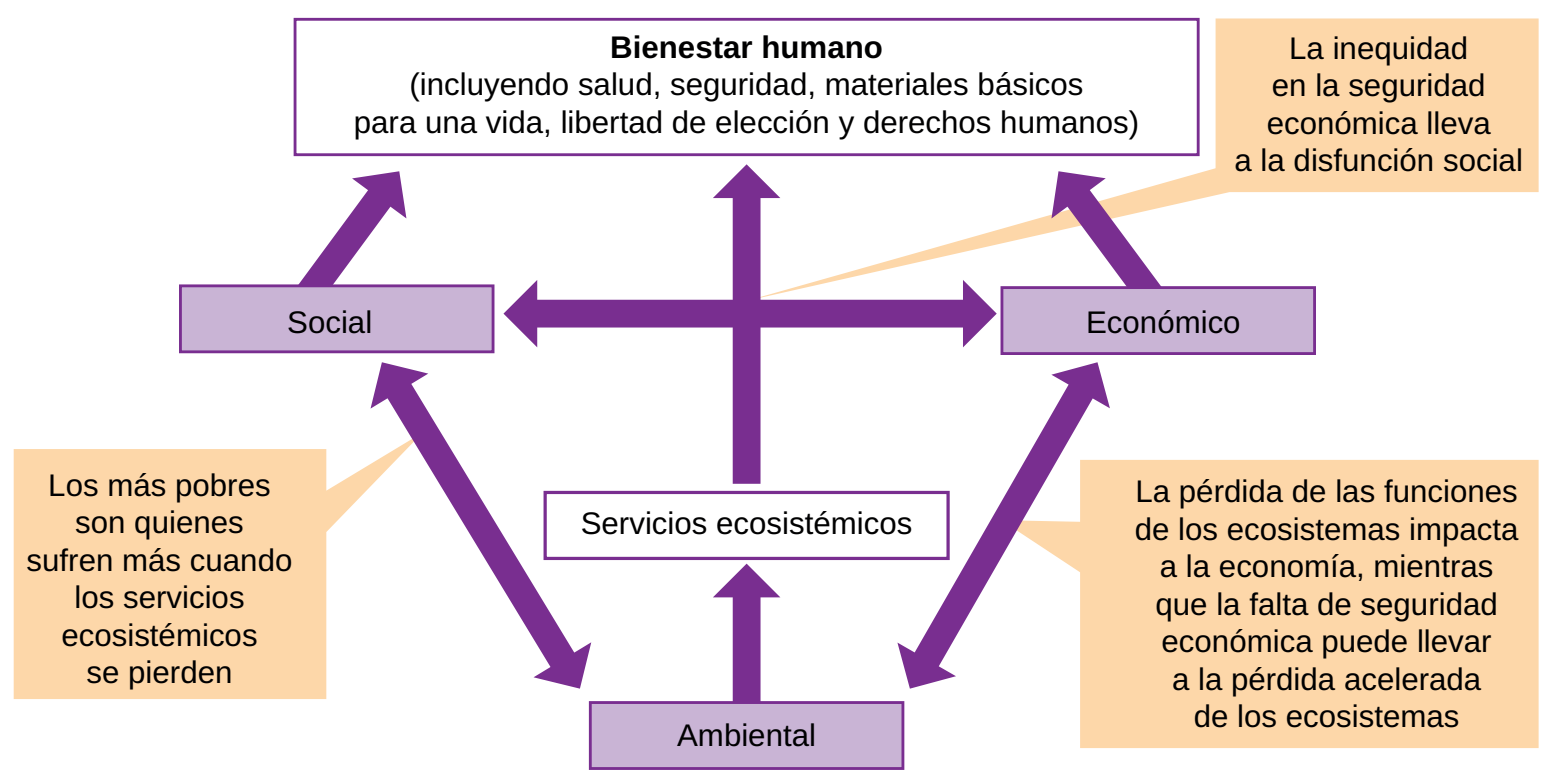

Fuente: United Nations Environment Programme [UNEP]. (2012). Programa de las Naciones Unidas para el Medio Ambiente.

Lo que advierte la ilustración anterior es una estrecha relación entre el desarrollo social y el económico en función de la satisfacción de niveles óptimos en la calidad de vida de los individuos, así como un desarrollo equilibrado entre los distintos sectores socioeconómicos que impida la pérdida de ecosistemas y servicios ambientales, en donde los que menos tienen regularmente son los que sufren los efectos negativos de este hecho (generación de asentamientos irregulares, falta de equipamiento y servicios, entre otros).

\section{Consideraciones del desarrollo territorial, urbano y arquitectónico actual en torno a la sustentabilidad}

$\mathrm{Al}$ analizar cada uno de los aspectos implícitos en la definición y compararlos con las condiciones de desarrollo de proyectos territoriales, urbanos y arquitectónicos actuales, se puede advertir que tal ejercicio no se ha cumplido a satisfacción. Un factor que advierte los efectos de entornos urbano-arquitectónicos alejados de principios de sustentabilidad es el cambio climático, que en los últimos años ha ocupado titulares y espacios importantes en medios masivos de comunicación como una de las principales preocupaciones de la comunidad científica internacional, así como de gobiernos de diferentes niveles, presionados por ONG relacionadas con la preservación medioambiental para el establecimiento de políticas públicas y prácticas que frenen y reviertan paulatinamente este proceso.

Otra causa, originaria de tal problemática, ha sido la transformación de los ecosistemas naturales en desarrollos urbano-arquitectónicos generadores de islas de calor, ocasionadas por las grandes cantidades de asfalto presente en vialidades, grandes superficies de estacionamientos de centros comerciales, disminución de áreas verdes, uso de materiales inadecuados en arquitectura acordes a la zona geográfica, y el uso excesivo del automóvil particular como medio de transporte, entre otros. ¿Qué hacer para revertir este proceso?

\section{El papel de las instituciones de educación superior en el cumplimiento de la sustentabilidad}

Un papel protagónico, acorde a los componentes de la sustentabilidad mencionados en este artículo, es el educativo y cultural, inmerso en los procesos sociales; donde las instituciones de educación superior tienen un compromiso y una responsabilidad directa para con su comunidad, en el sentido de la trasmisión de conocimientos y valores hacia sus educandos con relación a la inclusión del tema en los programas de estudio de sus carreras, particularmente en la de arquitectura, estableciendo metodologías claras y de fácil aplicación y comprensión para los estudiantes, así como una sencilla evaluación por parte de los docentes. 


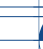

DOCERE Una visión sobre los principios de la sustentabilidad para la enseñanza de la arquitectura

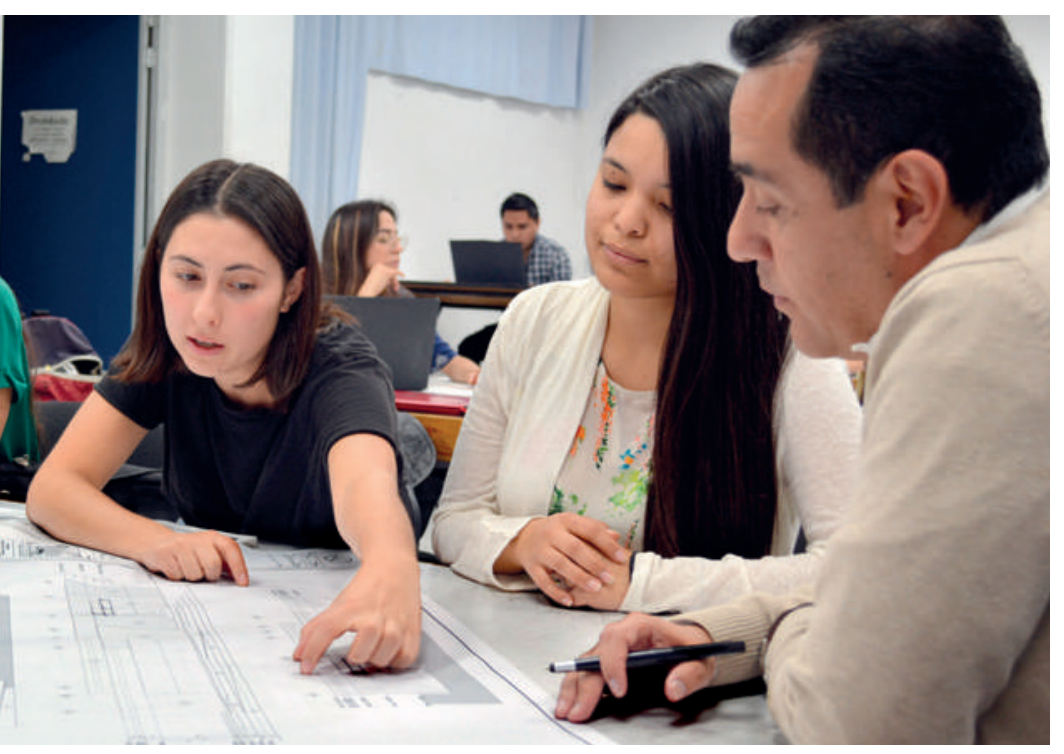

\section{Conclusión}

Para lograr que el desarrollo sustentable sea una realidad en nuestra vida cotidiana, es preciso que se forme e informe a la población, y en específico a los profesionales de la arquitectura desde las aulas, en donde, además de la inclusión de materias que aporten conocimiento en torno al tema, se trabaje con metodologías claras y medibles, que sean adoptadas en los trabajos de las academias como parte de la evaluación y seguimiento en los procesos de proyectos en los talleres de diseño arquitectónico, desde su concepción teórica e investigación, hasta la puesta en práctica en los modelos tridimensionales, acorde a nuestra realidad socioeconómica y geográfica.

Se considera pertinente discutir, diseñar e implementar propuestas propias de sistematización en la enseñanza de la sustentabilidad en arquitectura, a partir de modelos que ya se han puesto en marcha en instituciones de educación superior en donde se enseña la arquitectura; comprometiendo a los involucrados en los procesos de enseñanza y aprendizaje: desde las autoridades, la administración para la planeación y evaluación de los programas, el cuerpo docente, hasta los estudiantes; lograr el involucramiento colectivo en su correcta aplicación, persiguiendo, ante todo, el bien común y el de nuestro planeta.

\section{Fuentes de consulta}

Castillo, C. (2013). Plan de Sustentabilidad en la Arquitectura, Universidad Diego Portales.

Aunque el programa de estudios de la carrera de arquitectura contempla actualmente tres materias relacionadas directamente con la sustentabilidad (Arquitectura sustentable, Ecotecnologías y Arquitectura bioclimática), se precisa de una guía general que sirva como base para la evaluación de proyectos arquitectónicos basados en el modelo de la sustentabilidad, al mismo tiempo que sea aplicable, al menos, en los talleres de diseño arquitectónico.

En ese sentido, se puede mencionar la propuesta de un método de sistematización para la sustentabilidad en arquitectura, propuesto por la Escuela de Arquitectura de la Universidad Diego Portales de Chile, en colaboración con el Centro de Desarrollo Urbano Sustentable (CEDEUs) de la Universidad Católica de Chile. En esta propuesta, se plantean las dimensiones, elementos, temas y criterios a considerar en todo plan urbano-arquitectónico que permitan dar seguimiento puntual al proceso de desarrollo de un proyecto, tanto por el alumno como por el docente (ver Tabla 1).

Castillo, C. y Del Castillo, M. (2015). Enseñanza, sustentabilidad, Arquitectura. Arquitecturas del Sur, 30-43.

ONU (2018). Objetivos de Desarrollo. México: ONU. Consultado el 30 de julio de 2018, en: http://www.onu.org.mx/ agenda-2030/objetivos-de-desarrollo-del-milenio/.

SEDATU (2014). Guía metodológica para la elaboración de programas estatales de ordenamiento territorial. México: Secretaría de Desarrollo Agrario, Territorial y Urbano.

UAA (2018a). Centro de Ciencias del Diseño y de la Construcción. Aguascalientes, México: UAA. Consultado el 30 de julio de 2018, en: http://www.uaa.mx/centros/ccdc/.

UAA (2018b). Oferta Educativa Arquitectura. Aguascalientes, México: uaA. Consultado el 30 de julio de 2018, en: https://bit.ly/1SihstK.

United Nations Environment Programme (2012). Marco institucional para el desarrollo sostenible. Quito, Ecuador: UNEP-ONu. Recuperado de: https://bit. ly/2w83N5n. 
Tabla 1. Sistematización de la sustentabilidad

\begin{tabular}{|c|c|c|c|}
\hline Dimensión & Elementos & Temas & Criterios \\
\hline \multirow{20}{*}{$\begin{array}{l}\frac{\bar{J}}{\frac{\pi}{0}} \\
\frac{0}{0} \\
\frac{\varepsilon}{<}\end{array}$} & \multirow{4}{*}{ Tierra } & \multirow{2}{*}{ Topografía y geología } & Considera relieve natural \\
\hline & & & Considera tipo de suelo \\
\hline & & \multirow{2}{*}{ Materiales } & Materiales reciclados certificados \\
\hline & & & Materiales locales naturales \\
\hline & \multirow{4}{*}{ Agua } & \multirow{2}{*}{ Ahorro y captación } & Captación y acumulación de aguas \\
\hline & & & Dispositivos de ahorro hídrico \\
\hline & & \multirow{2}{*}{ Reutilización y reincorporación } & Reutilización de aguas \\
\hline & & & Reincorporación del ciclo hidrológico \\
\hline & \multirow{4}{*}{ Aire } & \multirow{2}{*}{ Acondicionamiento térmico } & Aislación pasiva, frío y calor \\
\hline & & & Calefacción, refrigeración activa y renovable \\
\hline & & \multirow{2}{*}{ Ventilación y humedad } & Ventilación natural \\
\hline & & & Ventilación artificial con renovable \\
\hline & \multirow{4}{*}{ Energía } & \multirow{2}{*}{ Orientación solar } & Emplazamiento y distribución programática \\
\hline & & & Iluminación natural y sombreado \\
\hline & & \multirow{2}{*}{ Eficiencia energética } & Ahorro, reducción en el consumo energético \\
\hline & & & Producción con energías renovables \\
\hline & \multirow{4}{*}{ Vida } & \multirow{2}{*}{ Habitabilidad } & Control y confort acústico \\
\hline & & & Tratamiento de residuos y control de emisiones \\
\hline & & \multirow{2}{*}{ Biodiversidad y paisaje } & Integración de la biodiversidad y al paisaje \\
\hline & & & Introducción de vegetación local \\
\hline \multirow{8}{*}{$\begin{array}{l}\overline{\frac{\pi}{U}} \\
\text { க) }\end{array}$} & \multirow{4}{*}{ Cohesión } & \multirow{2}{*}{ Identidad } & Historia, cultura y patrimonio \\
\hline & & & Seguridad, confianza e integración \\
\hline & & \multirow{2}{*}{ Gobernanza } & Gestión política y legislación \\
\hline & & & Participación y asociatividad \\
\hline & \multirow{4}{*}{ Movilidad } & \multirow{2}{*}{ Accesibilidad } & Accesibilidad universal \\
\hline & & & Accesibilidad a servicios y equipamientos \\
\hline & & \multirow{2}{*}{ Transportes } & Transporte público eficiente \\
\hline & & & Fomento del modo pie y bicicletas \\
\hline \multirow{8}{*}{ 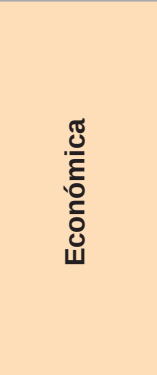 } & \multirow{4}{*}{ Conectividad } & \multirow{2}{*}{ Infraestructuras } & Optimización y diseño de la red de transporte \\
\hline & & & Distribución de energía \\
\hline & & \multirow{2}{*}{ Redes } & Sistemas digitales de comunicación \\
\hline & & & Cooperativismo \\
\hline & \multirow{4}{*}{ Eficiencia } & \multirow{2}{*}{ Nuevas economías } & Economía ecológica y empleos verdes \\
\hline & & & Economía local y servicios ambientales \\
\hline & & & Adaptación y creatividad \\
\hline & & minovacion & Vida útil eficiente con menos mantenimiento \\
\hline
\end{tabular}

Fuente: Castillo, C. (2013). 